have regarded it as a case of typhoid fever. There occurred, however, after the patient had been in the hospital four or five days eharacteristic typhoid stools. The reaction had been obtained in a very pronounced manner immediately upon the pa tient entering the hospital, which was on the third or fourth day of his illness. The course of the disease in that case was short, lasting only about two weeks. In other instances the reaction was not obtained until late in the disease. Of the cases examined not of typhoid fever, Dr. Miller found the reaction in 2.5 per cent. In part of these cases there was a history of prior typhoid fever, but not in all of them. It is evident, therefore that while this reaction is very frequently present in typhoid fever, it is not an absolutely constant occurrence.

I feel inclined to criticise the tendency to call the reaction the typhoid fever test. Would it not be better to call it a symptom of typhoid fever? We have other symptoms of typhoid fever upon which we have relied in times past very largely in making the differential diagnosis where diagnosis was difficult, for example the occurrence of rose spots. Yet we know that rose spots occur occasionally in other diseases than typhoid fever. We know they do not occur in all cases of typhoid fever. Yet when we find rose spots in connection with other symptoms we make our diagnosis. We rely upon the tout ensemble rather than upon the individual symptoms. Therefore, would it not be better to regard the serum reaction as a symptom of typhoid fever rather than as a test, and take it only in connection with other symptoms, and not look upon it so exclusively, as some seem inclined to do, as a test pure and simple for typhoid fever?

Dr. H. A. West, Galveston, Texas-I wish to make one remark with regard to the importance of this test in sections of the country from which I come. It appears to me that it may be the possible means of settling some vexed questions in my section of the country with regard to the diagnosis of fevers. While in my own mind I have been satisfied as to the extensive prevalence of typhoid fever throughout Texas, and believe that the continued fevers which fail to yield to the salts of cinchona are typhoid fever, yet there is a division of opinion and the question is far from being settled. There is a class of practitioners who do not regard this continued fever as typhoid. They do not see the eruption; they do not find the ordinary classical symptoms of typhoid fever; they call it slow fever, catarrhal fever, simple continued fever, thermic fever, etc. There is another class of practitioners who call them cases of continued malarial fever. While they acknowledge the fact that they last three or four weeks, sometimes six or eight weeks, in spite of daily doses of 20 to 40 grains of quinin given during that period, still it is claimed that they are cases of malarial fever. The question came up at the State Association, and I regard it as one of the most important questions before the South or the country at large. From what I have heard this evening, I do think this method may be the means of settling that vexed question. I propose to carry it out to the extent of my ability, but I may say that the cases which $I$ see in Galveston are classical typhoid fever with eruption, and running the course which typhoid fever runs the world over.

Dr. J. B. Herrick, Chicago-I will mention some of the results obtained in about one hundred cases by Dr. George $H$. Weaver in the laboratory of Rush Medical College. In general his results have been confirmatory of those we have listened to today, but in two cases, shown postmortem to be typhoid, the blood examination during life and after death was negative. Now it may be a peculiar coincidence that in these cases there was also tuberculosis. One patient, an aduit negro, under my own observation, died of typhoid fever as nearly as we can judge, in the fourth week, and postmortem we found typical healing typhoid but also a tubercular gland opening into a vein serving to explain distinct tubercular foci. In the second case, not under my own observation, a somewhat similar finding was made. This, I say, may be a coincidence, but it opens up the question whether this serum reaction will be modified by mixed or secondary infection. I believe other observers have reported on a fow cases of mixed infection where the reaction has been positive.

\section{RELAPSES IN TYPHOID FEVER.}

Presented in the Section on Practice of Medicine. at the Forty-eighth Annual Meeting of the American Medical Association,

held at Philadelphia, Pa., June 1-4, 1897 . BY WILLIAM OSLER, M.D. BALTIMORE, MD.

One of the most distressing incidents which we may meet in typhoid fever is the occurrence of a relapse after we have guided the case safely to convalescence. The temperature has become normal, the patient is happy and hungry, the relatives contented, the doctor congratulates himself that he has cured a case of typhoid fever, and then comes the relapse and the tempest of the soul, so to speak. In the first place it is always a source of congratulation to the doctor that he is not personally responsible for the relapse. The condition under which relapse occurs is not, I think, in the large majority of cases within the control of the physician. Errors in diet are in some instances held responsible for it, but only in a very limited number of cases can the diet be held to be definitely responsible.

It is important in the first place to define accurately what a relapse is. I think the term should be limited exclusively to reinfection after a distinct and definite period of apyrexia. It is quite true that that definition does not include a certain number of cases which have been termed the intercurrent relapse, and we have a certain number of cases in which the temperature falls not quite to normal, say to 100 degrees F. or the morning temperature to 99.5 degrees, while for three or four days there is a distinct lull and then a recurrence of the symptoms. One is often in doubt whether such a case should be positively called a relapse or not; but such cases should be grouped as intercurrent relapses and not as true relapses. The occurrence of a relapse is an indication that immunity in typhoid fever is slowly acquired, and not fully acquired when apyrexia is reached.

The sources of the reinfection are as yet unknown, just as are the conditions under which relapse develops; but of the possible sources of reinfection we may mention the lingering of the typhoid bacilli in the mucous membrane and in the adenoid tissues of the intestines. We have been told today by Dr. Richardson, that even after a considerable period of apyrexia typhoid bacilli have been found in the stools. Then it is possible that the source is in the mesenteric glands and the spleen--in the latter region we know that typhoid bacilli remain for a very considerable length of time after convalescence. Chiari recently made an interesting suggestion that the typhoid bacilli remain in the gall bladder, persisting after a long period of time, not only for weeks but even for months, and that the organisms in the gall bladder may be the source of reinfection. $\mathrm{He}$ has found typhoid bacilli in the gall bladder in nineteen out of twenty-three cases, a much larger percentage than has been met with by other observers. But the persistence of the typhoid bacilli in the bile passages is well recognized, and he suggests that the taking of an extra amount of food and gastric disturbance may be associated with the passage of a large number of these bacilli from the gall bladder into the intestines.

The evidence of relapse is very different in different series of cases. Up to Aug. 5, 1896, 500 cases of typhoid fever were treated in the wards of Johns Hopkins Hospital and forty cases had relapsed, or 8 per cent. That is the same percentage as is given by Liebermeister in about the same number of cases. It is a very much larger percentage than that given by Murchison in a larger series of cases - in two or three thousand cases there were only 3 per cent. of relapses. Other percentages run up as high as 20 , but older observations, and even more recent ones, show that the percentage of relapses varies very much indeed. The relapses may not be single but double, now and then triple, and a few cases have been reported in which four or five relapses occurred. We have only 
had a few cases in which two relapses occurred, none in which there were three.

As illustrating the extraordinary length to which a case of typhoid may drag itself with only two relapses, I may mention a case which was admitted in October, 1893. The patient was a doctor, admitted to a private ward with well characterized typhoid fever. The temperature became normal on the forty-first day. There was then complete apyrexia for twenty-three days and the patient had arranged with relatives to be taken home. But fever developed and persisted forty-one days, the temperature reaching from 104 to 105 degrees $\mathbf{F}$. Then there was a period of complete apyrexia for forty-two days, six full weeks without fever. Then there was fever for fourteen days, withont rose spots, it is true, but with furred tongue and moderately high fever and with some abdominal distension. So that altogether the original attack with the two relapses covered a period of very nearly six months.

The mode of onset of the relapse is interesting. It is not always, as stated in most works, exactly like the original attack--a stepping up of the temperature each day. It may, in fact, begin abruptly, as in the case which $I$ have just mentioned. The onset of the first relapse was with a series of chills, and a maximum temperature of 105.5 degrees was reached within twenty-four hours of the beginning of the fever. Those cases are not very uncommon. In other instances the relapse is the exact counterpart of the primary attack, the temperature rising gradually, a degree or a degree and a half daily.

Another question has been raised to which I would like to refer, namely, whether the Brand treatment has anything to do with the increased number of relapses. Our percentage is, as you have noticed, not very high, and I do not think one can state that hydrotherapy has any influence in promoting relapses. There are many series of cases with a larger proportion of relapses in which hydrotherapy had not been carried out. Indeed, in certain series in which the relapses have been up to 15 or 20 per cent., the ordidary expectant method of treatment, or perhaps more correctly, the various medicinal plans of treatment have been carried out.

\section{FURTHER REPORT OF CASES TREATED WITH ANTI-TUBERCLE SERUM.}

Read in the Section on Practice of Medicine at the Forty-eighth Annual Meeting of the American Medical Association, held

at Philadelphia, Pa., June 1-4, 1897.

BY PAUL PAQUIN, M.D.

ST. LOUIS, MO.

In presenting this report to the AMERICAN MEDICAL Association I am not mindless of the many objections which have been presented against sero-therapy in this disease, and of the numerous criticisms which have been passed upon it, and of the feeling of apprehension and doubt which still pervades the minds of many of the profession. I am fully aware that experimental tuberculosis in small animals has failed to accomplish to the degree expected, the kind of socalled cure, hoped for. Nevertheless, I again take the liberty of presenting a report of cases treated with serum, with the results obtained since the last months of 1894 to this date. Naturally, I must leave out a large number of cases of which the reports are incomplete, or otherwise unsatisfactory for the reason, on the one hand, that some physicians did not keep the proper records, and on the other, there was in certain cases some doubt of the nature of the disease and of the results obtained. The cases reported here are such as I know had tuberculosis, and, I feel convinced, have experienced the results I shall mention.

In January, 1895, I presented to the St. Louis Medical Society a report of the results obtained in twentytwo St. Louis City Hospital patients, who had been treated exclusively with serum for three months. The good results in certain classes of patients were so uniform that the presentation of the matter was warranted at the time. The experiments were interrupted there by change of administration and the patients scattering I was able to keep track of only three of those improved, all of whom are still living and back at work apparently well, although I do not yet use the designation cured.

A second report of entirely new cases was made to the St. Louis Medical Society in March, 1896, and included the recovery of two acute cases of pulmonary tuberculosis, who had been in the care of three lead. ing physicians of Missouri, who bad given as their opinion that there was no hope. It also included the recovery of fourteen cases of chronic pulmonary tuberculosis. In that report I made the following statement: "That of 100 cases treated in a period of eighteen months, the following results obtäined: In the first and second stage, as understood in general practice, 14 recovered; in the second stage, improved or checked or still improving at the time, 26; among these 26,12 seemed to have really recovered, although the time had not been long enough to warrant positive assurance; there had disappeared from observation, in all stages, 35; died during treatment, begun at the third and fourth stages, including 10 moribunds who insisted on trying the treatment, 25. In the same year, Dr. Hayden of Evansville, Ind,, reported 4 cases of tuberculosis; all improved and 2 apparently recovered. Besides these. Drs. L. L. Shropshire, San Antonio, Texas; Dr. William Miller, Boerne, Texas; Dr. Hoell Tyler, Mentone, Cal.; Dr. H. S. Lewis, New Orleans, La.; Dr. E. L. Mitchell, Carbondale, Ill.; Dr. J. M. Allen, Liberty, Mo.; Dr. A. G. Deardorff, San Francisco, Cal.; Dr. J. L. Wiggins, E. St. Louis, Ill.; Dr. J. L. Dunwoody, Cripple Creek, Colo.; Dr. W. H. Prioleạu, Summerville, S. C., reported the recovery of ever twenty-five cases and marked improvement in over one hundred, while at least seventy-five physicians have reported improvements, of various degrees, in over one hundred cases impossible to record, most of them hopeless from the beginning.

In September, 1896, I presented a report to the Virginia State Medical Society, of a total of 226 cases of tuberculosis. I quote from this paper:

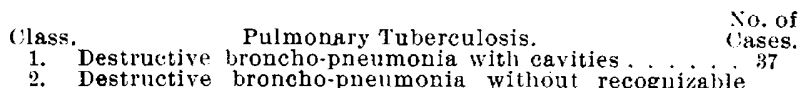

$$
\begin{aligned}
& \text { 2. Destructive broncho-pneumonia without recoguicable } \\
& \text { 3. With diffuse febrile pneumonia with or without a destruc- } \\
& \text { With diffuse febrile pneumonia with or without a destruc- } \\
& \text { With diffuse non-febrile broncho-pneumonia with or with. } \\
& \text { out destructive cavities } \\
& \text { With cfreumseribed febrile brovelio-pneumonia } \\
& \text { With circunseribed apyretic broucho-pneumonia. } \\
& \text { Diagnosis not reported clear enough for classification } \\
& \text { Hip-joint tubereulosis } \\
& \text { ovarian tuberculosis. }
\end{aligned}
$$

In every one of these cases the diagnosis had been verified microscopically. During the treatment of these 226 cases the following conditions obtained: 